\title{
Technologische Innovationen für das Monitoring im integrativen Flussbau
}

Das vorliegende Sonderheft ist als thematische Erweiterung zu den bereits erschienenen Veröffentlichungen in der Ausgabe 3-4/2020 zu sehen. Innovationen in Form von neuen Technologien und Methoden sind im Wasserbau jene zukunftsweisenden Felder, welche zielgerichtet Investitionen in die technische Optimierung von Anlagen bzw. auch in ökologische Verbesserungen im Sinne eines integrativen Fließgewässermanagements ermöglichen.

Einer jener Schwerpunkte bezieht sich auf die Optimierung von Fischaufstiegshilfen (FAHs), wo das Thema Auffindbarkeit nach wie vor eine Reihe unbeantworteter Forschungsfragen hinsichtlich der Instinktbewegung von Fischen, ausgelöst durch Schlüsselreize, aufwirft. Die Auffindbarkeit von FAHs wird in verschiedenen Leitfäden, neben der Positionierung der Lage des Einstiegs, primär am Parameter Strömung (Rheotaxis) festgemacht. Aus hydraulischer Sicht hat jedoch die am Einstieg austretende Strömung schon von der Größe des Ausbreitungsfeldes her lediglich eine untergeordnete Wirkung. In Brandl et al. (2020) wird die systematische Abklärung der Wirkung alternativer Schlüsselreize auf die Auffindbarkeit von Fischaufstiegshilfen im Rahmen eines mehrstufigen Forschungsprojekts der VERBUND Hydro Power GmbH durchgeführt. Neben den Reizen in Form der verschiedenen Strömungsparameter wurden künstliche Reize, welche auf die Phonotaxis, also die Orientierung aufgrund akustischer Reize, und die Phototaxis, die Orientierung an Lichtreizen abzielen, gesetzt.

In Heine et al. (2020) werden neue Methoden der reflexionsseismischen Messungen in Fließgewässern und Stauräumen beschrieben. Informationen über den Aufbau der oberen Sedimentschichten sind sowohl für aktuelle Monitoring-Maßnahmen als auch für prognostische Prozessmodellierungen des Sedimenttransports von großer Bedeutung. Seismische Verfahren wie Sub-Bottom-Profiler (SBP) bieten hier eine sehr gute Möglichkeit zur Erfassung dieser Grundlagendaten. Ihre Anwendbarkeit ist jedoch eine Frage der vorliegenden Objekteigenschaften (Wassertiefe, Strömungsgeschwindigkeiten etc.) und die Qualität der Ergebnisse, wie Eindringtiefe und Auflösung der Sedimentstrukturen, wird stark von systemspezifischen Parametern und Sedimentbeschaffenheit wie Korngrößen beeinflusst. Der Beitrag von Heine et al. (2020) behandelt den Einsatz von zwei hochauflösenden und kompakten Seeseismik-Systemen zur Detektion des Sedimentaufbaus in Gewässern mit grundsätzlich unterschiedlichem Sohlaufbau. Ein Vergleich von Sedimentkernauswertungen mit den resultierenden Echogrammen lässt auf das große Potenzial von SBP bei Feinsedimentböden schließen, Analysen zeigen allerdings auch Anwendungsgrenzen im Kiesbereich auf.

Eine weitere Technologie, die bereits vielfältige Anwendung in der integrativen Wasserwirtschaft findet, ist die sogenannte Hydroakustik, die mittels Einsatz von Echoloten und Sonaren in Binnengewässern als eine etablierte Methode anzusehen ist. In Schmidt \& Schletterer (2020) wird diese um den Aspekt der Analyse von Fischbeständen und Fischverhalten erweitert. Bei der Aufarbeitung verschiedener Fallstudien aus Österreich wurde festgehalten, dass je nach Fragestellung vier unterschiedliche Systeme zum Einsatz kommen können: (1) Single-beam-Echolot, (2) Dual-beam-Echolot (Kombination von hohen und niedrigen Frequenzbereichen), (3) Split-beam-Echolote, welche die Erfassung von räumlichen und zeitlichen Fischverteilungen und Fischbiomasseerhebungen ermöglichen und (4) Multibeam-Sonare, sogenannte hydroakustische Kameras, welche die Analyse von Fischverhalten in tiefen und dunklen Gewässern ermöglichen. Der Artikel von Schmidt \& Schletterer (2020) beinhaltet Anwendungsbeispiele aus Österreich zum Einsatz hydroakustischer Methoden zur Fischdetektion, Fischbiomassebestimmung und zur Analyse von Fischverhalten. In dieser Arbeit wird aufgezeigt, dass hydroakustische Verfahren sowohl angewandt-wissenschaftlich als auch in der Grundlagenforschung erfolgreich eingesetzt und für aktuelle Fragen in Forschung und Praxis genutzt werden können. 


\section{Editorial}

Einen strategisch innovativen Ansatz im Management von Fließgewässern beinhaltet der Artikel von Zauner et al. (2020). In diesem Fachbeitrag wird die Frage nach der Erreichung des „guten ökologischen Potenzials“ gestellt und anhand des Fallbeispiels Innstauraum Egglfing-Obernberg präsentiert. Für die Erreichung dieses Ziels ist ein gemäß „Leitfaden zur Bewertung erheblich veränderter Gewässer" definierter Teil des „höchsten ökologischen Potenzials“ umzusetzen, worunter das maximale Maßnahmenpotenzial ohne signifikante Einschränkungen der für die Einstufung als „erheblich veränderter Wasserkörper" verantwortlichen Nutzungen zu verstehen ist. Für den Innstauraum Egglfing-Obernberg wurden die dazu erforderlichen Maßnahmen definiert und im Rahmen des von 2017 bis 2019 laufenden Projekts „Durchgängigkeit und Lebensraum“ großteils bereits umgesetzt. Unterschiedlichste Maßnahmen wurden hierbei konzipiert wie (i) die Herstellung eines Insel-Nebenarmsystems einschließlich eines unterstromig angebundenen Stillgewässers in der Stauwurzel, (ii) die Dynamisierung und Entlandung bestehender Altarme sowie (iii) die Errichtung eines naturnahen Umgehungsgewässers als Fischaufstiegshilfe und hochwertiger Fließgewässerlebensraum. Im Rahmen des über die nächsten Jahre laufenden Monitorings soll die Wirkung der Maßnahmen in Hinblick auf die Zielerreichung nach Wasserrahmenrichtlinie untersucht werden, wobei in Analogie zu den Ergebnissen bestehender fischökologischer Studien eine deutliche Stärkung der Fischpopulationen zu erwarten ist.

Die vier Beiträge dieses Sonderhefts zeigen in Ergänzung zur Ausgabe 03-04/2020 deutlich, dass sich unterschiedlichste innovative Technologien und Methoden bzw. auch Konzepte bereits national und international in erweiterten Testphasen befinden, indem in Forschungs- und angewandten Projekten Möglichkeiten und Grenzen ihrer Anwendung ausgelotet werden. Dadurch wird gewährleistet, dass auch in Zukunft weitere und auch notwendige Schritte hinsichtlich einer hydromorphologischen Verbesserung und integrativen Fließgewässersanierung in Österreich möglich sind bzw. Investitionen in die Zukunft der Gewässer zielgerichtet erfolgen können.

\section{PD DI Dr. C. Hauer (ه)}

\section{Univ.-Prof. DI Dr. H. Habersack}

Christian Doppler Labor für

Sedimentforschung und -management,

Institut für Wasserbau, Hydraulik und

Fließgewässerforschung, Universität für

Bodenkultur Wien,

Muthgasse 107,

1190 Wien, Österreich

christoph.hauer@boku.ac.at

Univ.-Prof. DI Dr. H. Habersack

helmut.habersack@boku.ac.at 